\title{
Chaotic mixing in microdroplets
}

\section{Roman O. Grigoriev, ${ }^{* a}$ Michael F. Schatz ${ }^{a}$ and Vivek Sharma ${ }^{b}$}

\author{
Received 17th May 2006, Accepted 10th July 2006 \\ First published as an Advance Article on the web 31st July 2006 \\ DOI: $10.1039 / \mathrm{b} 607003 \mathrm{e}$
}

\begin{abstract}
We describe a general methodology for introducing thorough chaotic mixing in microdroplets. The mixing properties of fluid flows in microdroplets are governed by their symmetries, which give rise to invariant surfaces serving as barriers to transport. Complete three-dimensional mixing by chaotic advection requires destruction of all flow invariants. To illustrate this idea, we demonstrate that complete mixing can be obtained in a time-dependent flow produced by moving a microdroplet along a two-dimensional path. The theoretical predictions are confirmed by experiments that use the thermocapillary effect to manipulate microdroplets.
\end{abstract}

\section{Introduction}

It is well known that liquids do not mix easily in microfluidic systems, which are being developed into "labs-on-a-chip" that promise revolutionary applications in biotechnology, chemistry and medicine. ${ }^{1-4}$ For devices based on continuous flow through microchannels, strategies for inducing chaotic mixing by altering device geometries have been proposed., Here we study mixing in discrete volume (microdroplet) systems, ${ }^{4,73}$ which allow miniaturization of many standard laboratory protocols that are difficult to realize with continuous flow.

Physically, typical microscale flows are characterized by a low Reynolds number $R e \equiv V a l v<1$, where $V$ and $a$ are, respectively, a characteristic flow speed and length, and $v$ is the liquids' kinematic viscosity. In this regime, flows are smooth (laminar), and turbulence, which dominates fluid mixing in standard-size laboratory tests, cannot arise. Mixing by molecular diffusion alone is often too slow, even at the scale of typical microfluidic devices. Thus, efficient mixing of liquids at the microscale requires a stirring mechanism, such as chaotic advection, ${ }^{14,15}$ that stretches and folds fluid elements throughout the entire volume of the flow. The folding leads to a decrease in the average distance between unmixed volumes of liquid with different composition, while stretching exponentially sharpens the concentration gradients enhancing diffusion, which acts more rapidly to smooth out nonuniformities.

Designing a chaotic microflow with good mixing properties is particularly difficult for highly symmetric flows such as those that arise in microdroplets. The high degree of symmetry leads to the existence of invariants, ${ }^{16}$ which are functions that are constant along streamlines of the flow. Each invariant defines, inside the volume of droplet, surfaces on which the flow is effectively two-dimensional. Additional invariants

${ }^{a}$ Center for Nonlinear Science and School of Physics, Georgia Institute of Technology, Atlanta, GA 30332-0430, USA.

E-mail: roman.grigoriev@physics.gatech.edu

${ }^{b}$ School of Polymer, Textile and Fiber Engineering, Georgia Institute of Technology, Atlanta, GA 30332-0295, USA further reduce the flow dimensionality; e.g., flow with two invariants is effectively one-dimensional. Since the flow cannot cross invariant surfaces, the existence of invariants is highly undesirable in the mixing problem as their presence inhibits complete stirring of the full microdroplet volume by chaotic advection. Thus, the key to achieving effective chaotic mixing in a microdroplet (indeed, in any laminar microflow) is to ensure that all flow invariants are destroyed.

\section{Theory}

To illustrate this, consider a spherical microdroplet immersed in another liquid and subjected to a temperature gradient, which induces surface tension gradients (thermocapillary effect) at the droplet interface. With a uniform temperature gradient (e.g., $\mathrm{d} T / \mathrm{d} x=\kappa_{0}=$ constant), the thermocapillary effect drives an axially symmetric flow along the surface from the warm pole to the cold one and a return flow along the axis of the droplet, which is pushed in the direction of the gradient. By solving the Stokes equation subject to the corresponding boundary conditions, the velocity field of this dipole-like flow, known as Hill's spherical vortex, ${ }^{17}$ is obtained,

$$
v_{x}=V \frac{a^{2}-x^{2}-2 y^{2}-2 z^{2}}{a^{2}}, v_{y}=V \frac{x y}{a^{2}}, v_{z}=V \frac{x z}{a^{2}},
$$

where $V=\kappa_{0} a \partial_{T} \sigma /\left(2 \mu_{\mathrm{o}}+3 \mu_{\mathrm{i}}\right), a$ is the radius of the droplet, $\sigma$ is the surface tension and $\mu_{\mathrm{i}}, \mu_{\mathrm{o}}$ are the dynamic viscosities of the liquid inside and outside the droplet.

This flow is effectively one-dimensional (and, therefore, non-chaotic) because it possesses two invariants

$$
I_{0}=\frac{z}{y} \text { and } J_{0}=z^{2}\left(a^{2}-x^{2}-y^{2}-z^{2}\right)
$$

related to the orientation of a plane containing the streamline and the stream function of the flow in that plane. This steady dipole flow has extremely poor mixing properties, as our experimental results (described below) illustrate. To achieve complete mixing, both invariants must be destroyed. One generic method to do so is by introducing time-dependence into the flow. ${ }^{14}$ For instance, time-periodic switching of the 

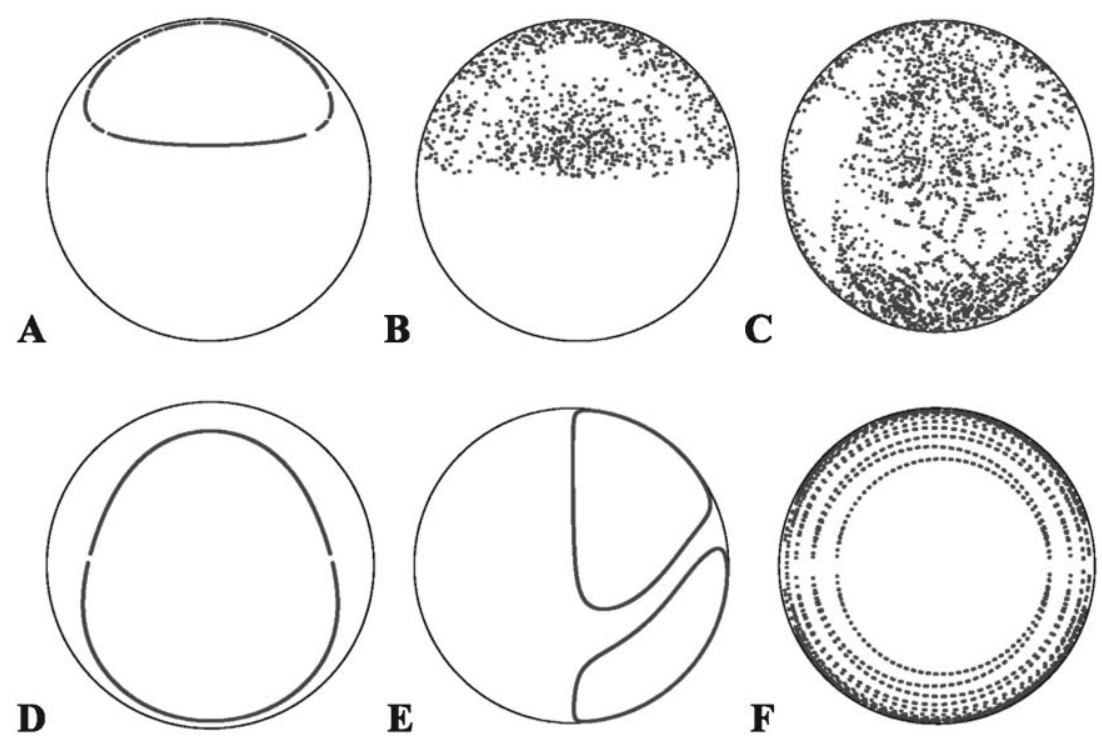

Fig. 1 Intersections of a dye particle's trajectory with the $x=0$ mid-plane of a spherical microdroplet illustrate mixing behavior. Unless noted otherwise, $\mu_{\mathrm{i}} / \mu_{\mathrm{o}}=1$ and the flow is made time-periodic by switching the direction of droplet motion in the horizontal plane by $90^{\circ}$ every 12 time units. The quadrupole flow is weak compared to the dipole component, $|\varepsilon|=0.4$ while the magnitude of the Taylor flow is comparable to that of the dipole flow. (A) Pure dipole flow. (B) Superposition of dipole and quadrupole flow. (C) Superposition of dipole, quadrupole, and Taylor flow. (D) Superposition of dipole and Taylor flow. (E) Superposition of steady dipole, quadrupole, and Taylor flow. (F) Superposition of steady dipole, quadrupole, and Taylor flows with $\mu_{\mathrm{i}} / \mu_{\mathrm{o}}=100$.

temperature gradient's direction in a plane (chosen to be the $(x, y)$-plane in this example) will cause the microdroplet to move in two dimensions and will lead to the switching of the axis of the dipole flow. The addition of direction switching breaks one of the invariants $\left(I_{0}\right)$ thus making the dynamics effectively three-dimensional (two space variables plus time) and introduces a positive Lyapunov exponent, which indicates that the time-periodic dipole flow is chaotic.

The presence of chaotic advection however does not guarantee complete mixing. Indeed, the invariant $J_{0}$ is preserved, so that chaotic flow is confined to two-dimensional surfaces of revolution inside the droplet given by

$$
x^{2}+y^{2}=a^{2}-z^{2}-\frac{J_{0}}{z^{2}}
$$

In a typical experiment, dye is used to monitor the mixing process. The flow (1) will only spread the dye over thin shells (see Fig. 1A) defined by eqn (3), where $J_{0}$ varies over the values corresponding to the initially dyed region of the fluid. ${ }^{18}$ Viewed from the top, a nearly uniform two-dimensional projection of the dye distribution in this case would misleadingly suggest thorough mixing. The same conclusion applies if the axis of the flow is rotated in a plane continuously or discretely by arbitrary angles at arbitrary times. Both invariants can, in principle, be destroyed by forcing the axis of the flow to move on the surface of a cone. ${ }^{19}$ However, this requires moving the droplet in three dimensions, which is much harder to achieve experimentally.

When the thermocapillary-driven droplet is restricted to move on a two-dimensional plane, a spatially nonuniform temperature gradient is required to destroy both flow invariants. Nonuniformity in the temperature gradient is modeled most simply by adding linear dependence (e.g., $\left.\mathrm{d} T / \mathrm{d} x=\kappa_{0}+\kappa_{1} x\right)$; the additional term induces a steady quadrupole-like flow of the same form as that caused by a linear axisymmetric extensional flow, ${ }^{18}$ and can be superimposed on the dipole flow because of the linearity of the Stokes equation. In the time-independent case, the flow is still axisymmetric and so also possesses two invariants: $\dagger$

$$
I=I_{0} \text { and } J=(1+\varepsilon x / a) J_{0}
$$

where $\varepsilon=\kappa_{1} a\left(2 \mu_{\mathrm{o}}+3 \mu_{i}\right) / \kappa_{0}\left(5 \mu_{\mathrm{o}}+5 \mu_{\mathrm{i}}\right)$. With the addition of time-periodicity induced by switching the direction of the temperature gradient, both flow invariants are destroyed for almost all values of $I$ and $J$; chaotic trajectories of the combined dipole/quadrupole flow are no longer confined to two-dimensional surfaces and explore a large fraction of the droplet volume (Fig. 1B).

A final transport barrier persists, preventing mixing between the top and bottom hemisphere of the microdroplet (Fig. 1B). This invariant surface, which is the mid-plane $z=0$ and corresponds to vanishing $I$ and $J, \$$ can be destroyed by imposing a uniform shear flow in the fluid surrounding the droplet with vorticity perpendicular to both the direction of the temperature gradient and the $z$ axis. This shear flow induces an internal circulation (Taylor flow ${ }^{21}$ ) that causes the streamlines to cross the $z=0$ midplane and enables essentially complete mixing ${ }^{22,23}$ (Fig. 1C).

\footnotetext{
$\dagger$ The expression (4) for $J$ shows that if the quadrupole component is sufficiently strong, i.e., $|\varepsilon|>1$. an additional invariant surface $x=-a / \varepsilon$ appears inside the droplet. This transport barrier can be destroyed by making $\varepsilon$ time-dependent. ${ }^{20}$ In this case, however, the invariant $I$ persists.

$\ddagger J$ also vanishes at the microdroplet's surface $x^{2}+y^{2}+z^{2}=a^{2}$, thereby representing a barrier to transport into or out of the droplet.
} 
In summary, all of these ingredients (time-dependence in conjunction with significant dipole, quadrupole and Taylor flow components) are essential for rapid, complete mixing throughout the droplet volume ${ }^{18}$ (Fig. 1). If any of these ingredients is missing, mixing becomes incomplete. For instance, the superposition of steady dipole and Taylor flows alone leads to non-chaotic flow preserving two invariants ${ }^{24}$ while the addition of time-dependence destroys one of the invariants, again leading to chaotic mixing on two-dimensional surfaces (Fig. 1D). As a second example, the superposition of steady dipole, quadrupole and Taylor flows possesses an adiabatic flow invariant which, for small $|\varepsilon|$ representative of the experiments described below, is preserved with a very good precision, confining chaotic trajectories to two-dimensional toroidal shells of extremely small thickness (Fig. 1E).

\section{Experiments}

To test the theory, we conduct experiments that can naturally include all ingredients predicted to be necessary for thorough mixing. Droplets containing glycerol-water mixtures are suspended at the surface of a $4 \mathrm{~mm}$-deep immiscible liquid substrate (Fluorinert $(\mathrm{R})$ FC-70 with $v=0.14 \mathrm{~cm}^{2} \mathrm{~s}^{-1}$ and $\rho=$ $\left.1.9 \mathrm{~g} \mathrm{~cm}^{-3}\right)$. The droplets are driven along the substrate surface by temperature-induced surface tension gradients produced by heating from a low-power infrared beam from a $\mathrm{CO}_{2}$ laser. $^{25}$ By rapid rastering of the laser beam, surfacetension gradients may be imposed at will at any location on the surface of the substrate. In this way, time-dependent switching of the microdroplet's motion can be implemented. Since the heating from the laser beam is localized, the corresponding temperature gradients are spatially nonuniform and, therefore, the thermocapillary-driven flow inside the droplet will be a combination of dipole flow with quadrupole (and, perhaps, higher-order) corrections. The laser beam also heats the free surface of the substrate liquid and, thereby, induces a surfacetension-driven shear flow (with vorticity perpendicular to the direction of thermal gradient) that gives rise to the Taylor circulation correction to the flow inside the microdroplet. ${ }^{18}$ The proximity of the microdroplet to the substrate interface can lead to additional corrections. However, according to our analytical and numerical calculations these corrections do not reduce the symmetry of the flow and hence the number of invariants, so the essential results of the model described above should still apply.

All mixing experiments begin by merging a single microlitrescale undyed droplet with a single nanolitre-scale droplet containing $0.5 \mu \mathrm{m}$ diameter fluorescent microspheres, which serve as passive tracers. After merger, the microspheres are initially localized in a small region near the surface of the combined droplet (Fig. 2A). Without further manipulation, diffusion would govern mixing of the microspheres in the combined droplet; the timescale required for diffusive mixing is $\sim 100$ days (the microspheres' diffusion coefficient of $7 \times 10^{-10} \mathrm{~cm}^{2} \mathrm{~s}^{-1}$ is determined from the StokesEinstein equation). To examine the effect of flow on mixing, the combined droplet is driven along a prescribed path on the interface with an average characteristic speed of
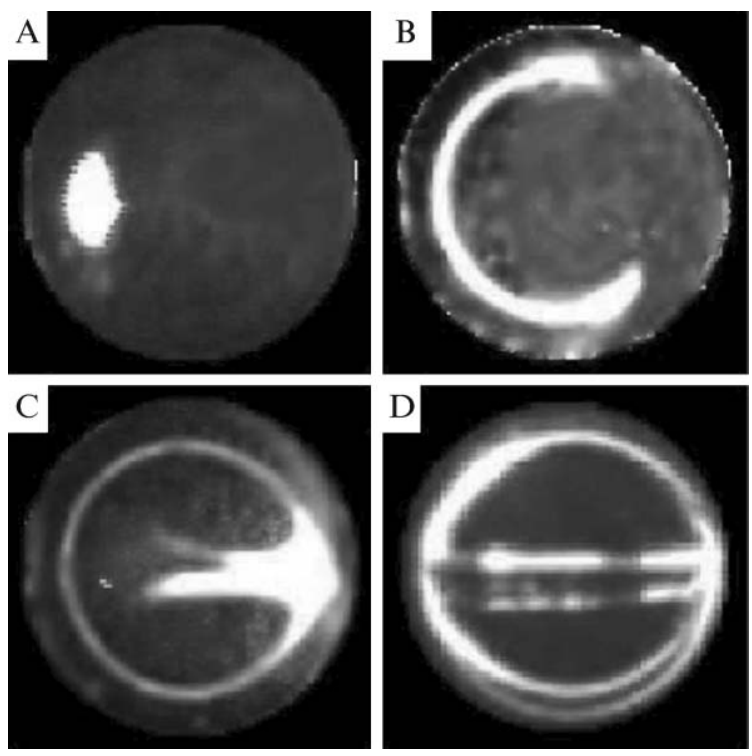

Fig. 2 Video images under monochromatic illumination at $488 \mathrm{~nm}$ illustrating optically controlled driving of a low viscosity (kinematic viscosity $0.12 \mathrm{~cm}^{2} \mathrm{~s}^{-1}$, corresponding to $\left.\mu_{\mathrm{i}} / \mu_{\mathrm{o}}=0.5\right)$ glycerolwater microdroplet in a single direction. (A) A 1 nanolitre droplet dyed with fluorescent microspheres is merged with a 14 nanolitre undyed droplet. The combined droplet is subsequently driven in one direction and imaged after traveling a distance of (B) 5 droplet diameters $(0.15 \mathrm{~cm}),(C) 8$ diameters $(0.24 \mathrm{~cm})$ and (D) 100 diameters $(3.0 \mathrm{~cm})$.

$U \approx 0.1 \mathrm{~cm} \mathrm{~s}^{-1}$. (Higher droplet speeds of a few $\mathrm{cm} \mathrm{s}^{-1}$ are possible with this system.) As a result, the microdroplet flow is characterized by low Reynolds number $\sim 0.1$ and high Péclet number $\sim 10^{7}$, placing the system in a laminar flow regime where mixing is expected to be particularly difficult.

Controlling both the composition and the path of the droplet in the experiments permits selective testing of the qualitative predictions of the model. The dipole, quadrupole and Taylor flows are predicted to be significant in droplets whose viscosities are comparable to that of the liquid substrate. ${ }^{18}$ If such droplets are subsequently driven in a single direction (time-independent driving), the dye is only distributed over a two-dimensional shell inside the droplet (Fig 2B-D). Such poor mixing is consistent with our theoretical analysis predicting the existence of an adiabatic invariant. In contrast, when the driving is made timedependent by periodically changing directions using the same protocol employed in the model, the dye is advected throughout the entire droplet volume (Fig. 3A and B). For a small number of direction changes, the droplet is thoroughly mixed, as predicted by the model. Complete three-dimensional mixing is verified experimentally by observation of the nearly uniform microsphere concentration at different planes within the droplet (Fig. 3C). The microsphere concentration is visualized by using a $50 \mu \mathrm{m}$-thick laser light sheet that is scanned across the entire droplet interior. As another independent test of the model, the dipole and quadrupole flow contributions are turned off experimentally by making the droplet viscosity much larger than the substrate viscosity. In 

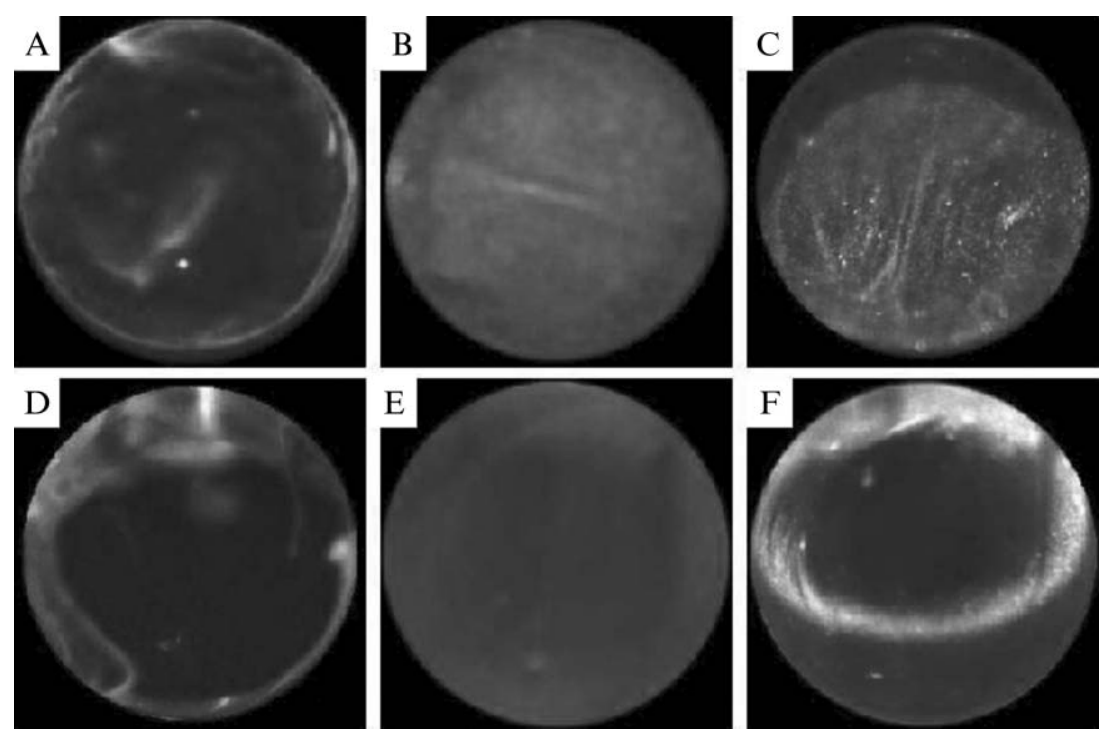

Fig. 3 Video images illustrating optically controlled driving of 200 nanolitre glycerol-water droplets along a closed square path. Mixing of a low viscosity $\left(\mu_{\mathrm{i}} / \mu_{\mathrm{o}}=0.4\right)$ droplet progresses rapidly as the droplet's total path length increases from (A) 13 droplet diameters $(0.9 \mathrm{~cm})$ to (B) 39 diameters $(2.7 \mathrm{~cm})$. Laser light sheet visualization of the droplet's interior $(\mathrm{C})$ verifies that mixing distributes passive tracers (fluorescent microspheres) uniformly throughout the droplet. By contrast, mixing of a high viscosity droplet (kinematic viscosity in the range of $2 \mathrm{~cm}^{2} \mathrm{~s}^{-1}$ corresponding to $\mu_{\mathrm{i} / \mu \mathrm{o}} \sim 10$ ) seems to progress well as the droplet's total path increases from (D) 13 droplet diameters $(0.9 \mathrm{~cm})$ to (E) 60 diameters $(4.2 \mathrm{~cm})$; however, laser light sheet illumination demonstrates that passive tracers are well mixed only in a thin 2-D shell (F) near the droplet's surface.

this regime, experiments suggest that the remaining ingredients of time-dependence via direction switching and Taylor flow lead to much poorer chaotic mixing, with the mixed region confined near the surface of the droplet (Fig. 3D-F), in accord with theoretical predictions (see Fig. 1F).

\section{Conclusion}

The analysis presented above should have broad applicability. On the most general level, one concludes that the existence and number of invariants play a crucial role in determining the mixing properties of the flow. In particular, in order to achieve full three-dimensional mixing the flow within the droplet should be designed to destroy all invariant surfaces in the interior of the droplet. More specifically, the types of flows that we have considered (dipole, quadrupole, and Taylor flow) are the most common types of interior flows arising inside spherical liquid microdroplets, regardless of the nature of driving forces, so many details of our analysis should be directly applicable to situations where microflows are driven by other effects such as external shear, buoyancy, or electrical fields. Finally, our results suggest that optically controlled thermocapillary actuation can be used as a general approach to performing many basic microfluidic operations such as moving, merging and mixing liquid microdroplets, opening up the road to a new generation of dynamically reprogrammable batch microfluidic devices.

\section{Acknowledgements}

Support for this work by the National Science Foundation and the American Chemical Society Petroleum Research Fund is gratefully acknowledged.

\section{References}

1 G. M. Whitesides and A. D. Stroock, Phys. Today, 2001, 54, 42-48.

2 L. Bousse, C. Cohen, T. Nikiforov, A. Chow, A. R. Kopf-Sill, R. Dubrow and J. W. Parce, Annu. Rev. Biophys. Biomol. Struct., 2000, 29, 155-181.

3 M. Burns, C. Mastrangelo, T. Sammarco, F. Man, J. Webster, B. Johnson, B. Foerster, D. Jones, Y. Fields, A. Kaiser and D. Burke, Proc. Natl. Acad. Sci. U. S. A., 1996, 93, 5556-5561.

4 T. S. Sammarco and M. A. Burns, AIChE J., 1999, 45, 350-366.

5 R. H. Liu, M. A. Stremler, K. V. Sharp, M. G. Olsen, J. G. Santiago, R. J. Adrian, H. Aref and D. J. Beebe, J. Microelectromech., 2000, 9, 190-197.

6 A. D. Stroock, S. K. W. Dertinger, A. Ajdari, I. Mezic, H. A. Stone and G. M. Whitesides, Science, 2002, 295, 647-651.

7 A. A. Darhuber, J. P. Valentino, J. M. Davis, S. M. Troian and S. Wagner, Appl. Phys. Lett., 2003, 82, 657-659.

8 P. Paik, V. K. Pamula, M. G. Pollack and R. B. Fair, Lab Chip, 2003, 3, 28-33.

9 J. Fowler, H. Moon and C.-J. Kim, Proc. IEEE Conf. MEMS, 2002, 97-100.

10 H. Song, J. D. Tice and R. F. Ismagilov, Angew. Chem., Int. Ed., 2003, 42, 768.

11 M. Muradoglu and H. A. Stone, Phys. Fluids, 2005, 17, 073305.

12 Z. B. Stone and H. A. Stone, Phys. Fluids, 2005, 17, 063103.

13 T. Ward and G. M. Homsy, Phys. Fluids, 2003, 15, 2987.

14 H. Aref, J. Fluid Mech., 1984, 143, 1-21.

15 J. M. Ottino, The Kinematics of Mixing: Stretching, Chaos, and Transport, Cambridge University Press, Cambridge, 1989.

16 I Mezic and S. Wiggins, Nonlinear Sci., 1994, 4, 157-194.

17 J. S. Hadamard, C. R. Acad. Sci. Paris, 1911, 152, 1735-1538.

18 R. O. Grigoriev, Phys. Fluids, 2005, 17, 033601.

19 J. R. Angilella and J. P. Brancher, Phys. Fluids, 2003, 15, 261-264.

20 T. Ward and G. M. Homsy, Phys. Fluids, 2001, 13, 3521-3525.

21 G. I. Taylor, Proc. R. Soc. London, Ser. A, 1932, 138, 41.

22 D. Kroujiline and H. A. Stone, Physica D, 1999, 130, 105-132.

23 A. I Neishtadt, D. L. Vainshtein and A. A. Vasiliev, Physica D, 1998, 111, 227-242.

24 M. D. Bryden and H. Brenner, J. Fluid Mech., 1999, 379, 319-331.

25 D. Semwogerere and M. F. Schatz, Phys. Rev. Lett., 2002, 88, 054501 . 